\title{
Assessing Parkinson's Disease From Speech Using Fisher Vectors
}

\author{
José Vicente Egas López ${ }^{1}$, Juan Rafael Orozco-Arroyave ${ }^{2,3}$, Gábor Gosztolya ${ }^{1,4}$ \\ ${ }^{1}$ Institute of Informatics, University of Szeged, Szeged, Hungary \\ ${ }^{2}$ Faculty of Engineering, University of Antioquia, Medellín, Colombia \\ ${ }^{3}$ Pattern Recognition Lab, University of Erlangen, Erlangen, Germany \\ ${ }^{4}$ MTA-SZTE Research Group on Artificial Intelligence, Szeged, Hungary \\ egasj a inf.u-szeged.edu
}

\begin{abstract}
Parkinson's Disease (PD) is a neuro-degenerative disorder that affects primarily the motor system of the body. Besides other functions, the subject's speech also deteriorates during the disease, which allows for a non-invasive way of automatic screening. In this study, we represent the utterances of subjects having PD and those of healthy controls by means of the Fisher Vector approach. This technique is very common in the area of image recognition, where it provides a representation of the local image descriptors via frequency and high order statistics. In the present work, we used four frame-level feature sets as the input of the FV method, and applied (linear) Support Vector Machines (SVM) for classifying the speech of subjects. We found that our approach offers superior performance compared to classification based on the i-vector and cosine distance approach, and it also provides an efficient combination of machine learning models trained on different feature sets or on different speaker tasks.
\end{abstract}

Index Terms: Parkinson's Disease, Fisher Vector encoding, speech analysis, automatic screening

\section{Introduction}

Shaking, rigidity, slowness of movement, and speech difficulties are some of the classic symptoms that affect the motor system, caused by a decrease of dopamine-producing neurons [1]. Such pathologies are often related to one of the most common neuro-degenerative disorders, that is, Parkinson's Disease. A person suffering from Parkinson's is prone to develop changes and disorders in speech and swallowing. This can occur at any time during the disease, but it generally appears as the disease advances. Commonly, the speech of the patient is also affected in terms of its tone, volume, and rate, which leads to dysprosody. Words comprising the speech of the subject may be slurred or mumbled. Additionally, typical articulatory problems exhibited by PD patients are referred to as dysarthia. Also, the speech can fade away at the end of the sentences; likewise, patients may speak slowly and with a breathy kind of speech $[1,2]$.

Utilizing Computer Tomography (CT) and Magnetic Resonance Imaging (MRI), the brain scans of people can be harnessed to diagnose PD. However, their results usually appear to be normal which makes it difficult for physicians to give an accurate diagnosis. Currently, there is no existing standard blood or laboratory tests that can be utilized to diagnose PD. Hence, the diagnosis, which sometimes may not be the most accurate, is often made based on the medical history of the patient and/or a neurological examination. In some cases, signs and symptoms of PD may be catalogued as the result of normal aging. Limitations within the commonly used process to assess patients with
PD include the high cost and the lack of efficiency when evaluating the disease. This process generally has two main drawbacks: it greatly depends on the expertise of the clinician, which is subjective; and the limitation of taking the patient to the clinic to try out exhaustive medical assessments and screenings [3].

There is a need to develop quick, reliable and non-invasive ways to diagnose PD. Thus, automatic speech analysis has been utilized in many medical branches in order to tackle the abovementioned obstacles by offering accurate and non-expensive solutions that are able to assess the diagnosis of different neurodegenerative diseases by the use of speech recordings. The most common scenarios include Alzheimer's [4, 5, 6] and Parkinson's Disease [7, 8], where the performance of different speech processing techniques such as i-vectors or ASR-based features (e.g. speech tempo or hesitation ratio) are applied.

Here, we will utilize the FV approach [9], which is an encoding method originally developed to represent images as gradients of a global generative GMM of low-level image descriptors. These new features are fed into a (linear) SVM [10] classifier in order to evaluate their capability to automatically discriminate between PD patients and Healthy Controls (HC). We will show that the proposed approach gives a better performance than for instance, using i-vectors, and provides a simple-yeteffective way of combining the predictions with other methods. To the best of our knowledge, this is the first study that focuses on making use of FV representation in order to detect speech impairments of PD patients.

\section{The Fisher Vector approach}

The Fisher Vector approach is an image representation that pools local image descriptors (e.g. SIFT, describing occurrences of rotation- and scale-invariant primitives [11]). In contrast with the Bag-of-Visual-Words (BoV, [12]) technique, it assigns a local descriptor to elements in a visual dictionary, obtained via a Gaussian Mixture Model for FV. Nevertheless, instead of just storing visual word occurrences, these representations take into account the difference between dictionary elements and pooled local features, and they store their statistics. A nice advantage of the $\mathrm{FV}$ representation is that, regardless of the number of local features (i.e. SIFT), it extracts a fixed-sized feature representation from each image.

The FV approach has been shown to be quite promising in image representation [9]. Despite the fact that just a handful of studies use FV in speech processing, e.g. for categorizing audio-signals as speech, music and others [13], for speaker verification $[14,15]$, and for determining the food type from eating sounds [16], we think that FV can be harnessed to improve classification performance in audio processing. 


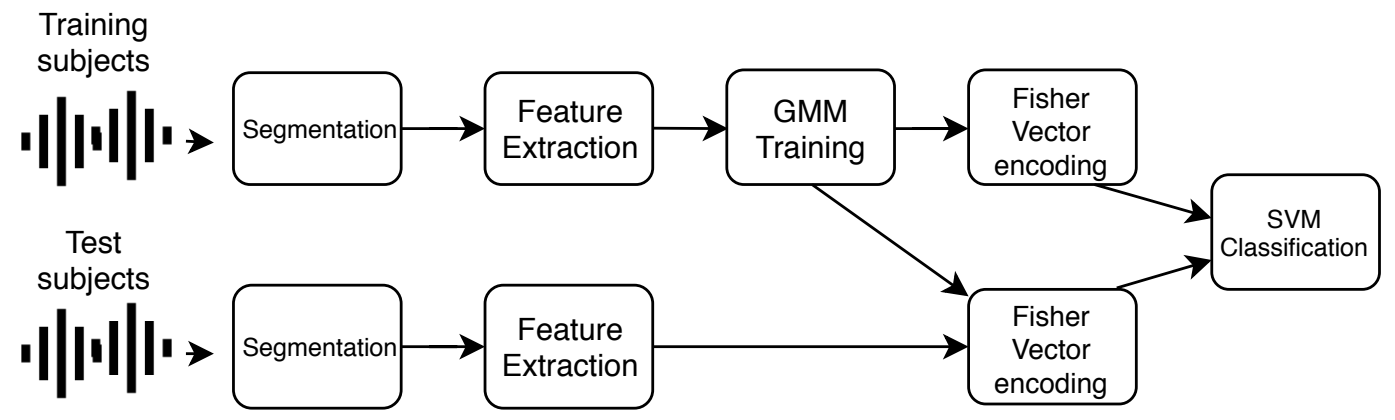

Figure 1: Generic methodology applied in our work.

\subsection{Fisher Kernel}

Named after the statistician Ronald Fisher [9], the Fisher Kernel (FK) seeks to measure the similarity of two objects from a parametric generative model of the data $(X)$ which is defined as the gradient of the log-likelihood of $X$ :

$$
G_{\lambda}^{X}=\nabla_{\lambda} \log v_{\lambda}(X)
$$

where $X=\left\{x_{t}, t=1, \ldots, T\right\}$ is a sample of $T$ observations $x_{t} \in \mathcal{X}, v$ represents a probability density function that models the generative process of the elements in $\mathcal{X}$ and $\lambda=\left[\lambda_{1}, \ldots, \lambda_{M}\right]^{\prime} \in R^{M}$ stands for the parameter vector $v_{\lambda}$ [17]. Thus, such a gradient describes the way the parameter $v_{\lambda}$ should be changed in order to best fit the data $X$. A novel way to measure the similarity between two points $X$ and $Y$ by means of the FK can be expressed as follows [9]:

$$
K_{F K}(X, Y)=G_{\lambda}^{X \prime} F_{\lambda}^{-1} G_{\lambda}^{Y}
$$

Since $F_{\lambda}$ is positive semi-definite, $F_{\lambda}=F_{\lambda}^{-1}$. Eq. (3) shows how the Cholesky decomposition $F_{\lambda}^{-1}=L_{\lambda}^{\prime} L_{\lambda}$ can be utilized to rewrite the Eq. (2) in terms of the dot product:

$$
K_{F K}(X, Y)=\mathscr{G}_{\lambda}^{X \prime} \mathscr{G}_{\lambda}^{Y}
$$

where

$$
\mathscr{G}_{\lambda}^{X}=L_{\lambda} G_{\lambda}^{X}=L_{\lambda} \nabla_{\lambda} \log v_{\lambda}(X) .
$$

Such a normalized gradient vector is the so-called Fisher Vector of $X$ [17]. Both the FV $\mathscr{G}_{\lambda}^{X}$ and the gradient vector $G_{\lambda}^{X}$ have the same dimension.

\subsection{Fisher Vectors}

Let $X=\left\{X_{t}, t=1 \ldots T\right\}$ be the set of $D$-dimensional local SIFT descriptors extracted from an image and let the assumption of independent samples hold, then Eq. (4) becomes:

$$
\mathscr{G}_{\lambda}^{X}=\sum_{t=1}^{T} L_{\lambda} \nabla_{\lambda} \log v_{\lambda}\left(X_{t}\right) .
$$

The assumption of independence permits the FV to become a sum of normalized gradients statistics $L_{\lambda} \nabla_{\lambda} \log v_{\lambda}\left(x_{t}\right)$ calculated for each SIFT descriptor. That is:

$$
X_{t} \rightarrow \varphi_{F K}\left(X_{t}\right)=L_{\lambda} \nabla_{\lambda} \log v_{\lambda}\left(X_{t}\right),
$$

which describes an operation that can be thought of as a higher dimensional space embedding of the local descriptors $X_{t}$.

In simple terms, the FV approach extracts low-level local patch descriptors from the audio-signals' spectrogram. Then, with the use of a GMM with diagonal covariances we can model the distribution of the extracted features. The log-likelihood gradients of the features modeled by the parameters of such GMM are encoded through the FV [17]. This type of encoding stores the mean and covariance deviation vectors of the components $k$ that form the GMM together with the elements of the local feature descriptors. The image is represented by the concatenation of all the mean and the covariance vectors that gives a final vector of length $(2 D+1) N$, for $N$ quantization cells and $D$ dimensional descriptors $[17,18]$.

The FV approach can be compared with the traditional encoding method called BoV (Bag of Visual Words), and with a first order encoding method like VLAD (Vector of Locally Aggregated Descriptors). In practice, BoW and VLAD are outperformed by $\mathrm{FV}$ due to its second order encoding property of storing additional statistics between codewords and local feature descriptors [19]. Here, we use FV features to encode the MFCC features extracted from audio-signals of $\mathrm{HC}$ and PD subjects. FV allows us to give a complete representation of the sample set by encoding the count of occurrences and high order statistics associated with its distribution.

\section{System description}

The architecture designed in our study consists of the following parts: (1) VAD-based segmentation, (2) feature extraction, (3) fitting a GMM to the local image features, (4) construction of the (audio) word dictionary by means of the GMM, that is, the encoded FV that now represents the global descriptor of the original spectrum, and (5) SVM classification. (See Fig. 1).

\subsection{Data}

We performed our experiments using the PC-GITA speech corpus [20], which contains the recorded speech of 100 Colombian Spanish speakers (50 PD patients and $50 \mathrm{HC}$ ). All of the patients were evaluated by a neurologist. The subjects were asked to perform four different tasks during the recordings: six diadochokinetic (DDK) exercises (e.g. the repetition of the sequence of syllables /pa-ta-ka/), monologue speeches, text reading, and ten short sentences.

\subsection{Feature Extraction}

Following the study of [21], we performed our experiments using four different feature sets. The first consisted of 20 MFCCs, obtained from $30 \mathrm{~ms}$ wide windows; and the rest of the feature sets were built by articulation, phonation, and prosody, respectively. Before extracting the features we performed speech/nonspeech segmentation by means of Voice Activity Detection 
Table 1: Results obtained for the various tasks and feature sets

\begin{tabular}{l|l||c|c|c} 
Task & Features & Acc. & $F_{1}$ & AUC \\
\hline \hline \multirow{5}{*}{ DDK } & MFCC & $\mathbf{7 8 \%}$ & $\mathbf{0 . 7 8}$ & $\mathbf{0 . 8 3 4}$ \\
& Articulation & $70 \%$ & 0.70 & 0.782 \\
& Phon./Artic. & $62 \%$ & 0.62 & 0.737 \\
& Prosody & $62 \%$ & 0.62 & 0.666 \\
\hline \multirow{5}{*}{ Monologue } & MFCC & $\mathbf{8 0 \%}$ & $\mathbf{0 . 8 0}$ & $\mathbf{0 . 8 8 0}$ \\
& Articulation & $76 \%$ & 0.76 & 0.847 \\
& Phon./Artic. & $70 \%$ & 0.70 & 0.749 \\
& Prosody & $58 \%$ & 0.58 & 0.621 \\
\hline \multirow{5}{*}{ Read text } & MFCC & $76 \%$ & 0.76 & $\mathbf{0 . 8 4 8}$ \\
& Articulation & $\mathbf{8 0 \%}$ & $\mathbf{0 . 8 0}$ & $\mathbf{0 . 8 4 8}$ \\
& Phon./Artic. & $72 \%$ & 0.72 & 0.758 \\
& Prosody & $78 \%$ & 0.78 & 0.798 \\
\hline \multirow{5}{*}{ Sentences } & MFCC & $\mathbf{8 0 \%}$ & $\mathbf{0 . 8 0}$ & $\mathbf{0 . 8 9 1}$ \\
& Articulation & $76 \%$ & 0.76 & 0.834 \\
& Phon./Artic. & $76 \%$ & 0.76 & 0.804 \\
& Prosody & $62 \%$ & 0.62 & 0.684
\end{tabular}

(VAD), and also by voiced/unvoiced using the auto-correlation method from Praat [22]. For articulation evaluation, the first 22 Bark bands (BBE) in voiced/unvoiced and unvoiced/voiced transitions were treated as features [23]. Features obtained from phonation and articulation in voiced segments constitute a 14dimensional vector with $30 \mathrm{~ms}$ of windows analysis and $5 \mathrm{~ms}$ of time shift. These features contained log-energy, pitch $\left(F_{0}\right)$, first and second formants $\left(F_{1}, F_{2}\right)$ together with their first and second derivatives, Jitter and Shimmer. Prosody information was represented by means of the approach introduced in [24]; hence, we got a 13-dimensional feature vector formed by using the number of voiced frames per segment and the 12 coefficients. To construct the FV representation, we experimented with $N=2,4,8,16,32,64$ and 128 Gaussian components. We utilized the VLFeat library in order to get the fisher vectors [25].

\subsection{Classification}

SVM was utilized to classify audio-signals into the PD and HC class labels. SVM was found to be robust even with a large number of dimensions and it was shown to be efficient when used with FV $[17,26]$ due to it being a discriminative classifier that provides a flexible decision boundary. We used the libSVM implementation [27] with a linear kernel, as suggested in [9]; the $C$ complexity parameter was set in the range $10^{-5}, \ldots, 10^{1}$.

The PC-GITA dataset is not large enough to define separate train, development and test sets; so in order to avoid any form of peeking, we performed the experiments in a speakerindependent 10-fold nested cross-validation (CV) setting; each fold contained the utterances of $5 \mathrm{PD}$ and $5 \mathrm{HC}$ speakers. Classification was made by using the SVM model trained on 9 folds (i.e. 90 speakers) and to get the right meta-parameters, we performed another $C V$ over the 90 speakers of the training folds. After determining the optimal $N$ (number of Gaussian components for $\mathrm{FV}$ ) and $C$ (SVM complexity) meta-parameters, we trained a SVM model with the 90 speakers using these metaparameter values. This way, we obtained predictions for all speakers without relying on any kind of data or information about the given subject.

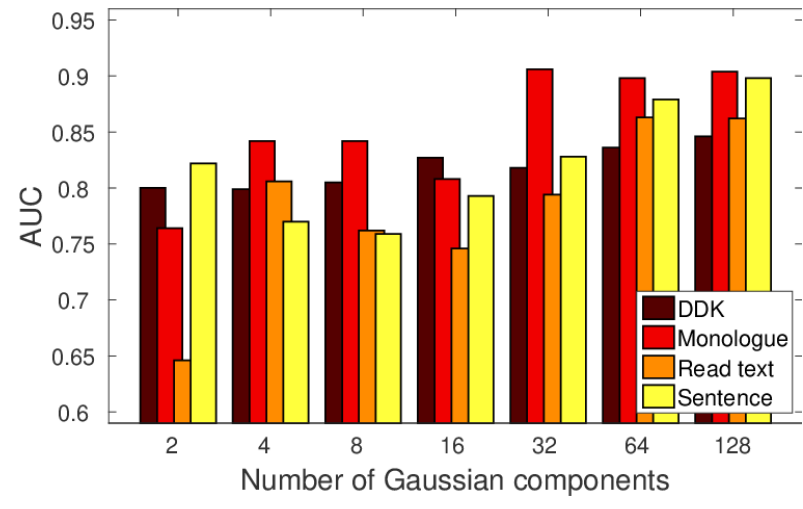

Figure 2: Achieved AUC values as a function of $N$ for the four speaker tasks, when using the MFCC feature set.

\subsection{Evaluation}

The decisions made by the SVM were used to calculate the Area Under the Receiver Operating Characteristics Curve (AUC), which is a widely used statistic for summarizing the performance of automatic classification systems in medical applications. In addition, we calculated the classification accuracy and F-measure (or $F_{1}$ ) scores. These metrics were calculated by choosing the decision threshold along with the Equal Error Rate (EER). Since the class distribution was balanced, classification accuracy and $F_{1}$ score were identical in each case. During the nested cross-validation procedure we determined the optimal meta-parameters as those that led to the highest AUC value.

\section{Results}

Table 1 lists the results we obtained for the different speaker tasks and the different frame-level feature sets, the best values for a given task being shown in bold. We observe that the best scores in each case were gotten with the MFCC feature set (except for the 'Read text' task, where the accuracy and $F_{1}$-scores appeared to be higher with articulatory features along with an identical AUC score). Although this was the case in our earlier studies as well (see [21]), where we relied on i-vectors, now the difference is significantly larger. This is probably because the FV approach assumed that the frame-level feature values could be modeled along with a diagonal covariance matrix. This assumption is quite realistic for MFCCs and, perhaps, for the filter bank values, of the voiced/unvoiced transitions (i.e. the articulatory features), but it may not be true for the phonational and prosodic attributes.

In the next experiment, we focused on the trends in the optimal number of Gaussian components (i.e. $N$ ) for the tasks. We tried out all the possible $N$ values, and then just the $C$ complexity parameter was determined in a nested CV. (Of course, this was not a completely fair setup from a machine learning perspective. Still, in our opinion, this small amount of 'peeking' was both necessary and acceptable in this scenario. Then we could focus on classification performance as a function of $N$.) Fig. 2 shows the AUC scores for the MFCC features. In general, using fewer GMMs $(N \leq 16)$ led to a sub-optimal performance, excepting the DDK task, where we can see a close-tooptimal AUC value even for $N=16$. For the Monologue task, $N=32$ components were needed for optimal performance, while $N=64$ and $N=128$ were enough for the Read text and the Sentences tasks, respectively. AUC scores were above 0.8 
Table 2: Results obtained when combining the different feature sets for the 'Monologue' task

\begin{tabular}{l||c|c|c} 
Features & Acc. & $F_{1}$ & AUC \\
\hline \hline MFCC & $80 \%$ & 0.80 & 0.880 \\
\hline MFCC + Articulation & $\mathbf{8 4 \%}$ & $\mathbf{0 . 8 4}$ & $\mathbf{0 . 9 0 8}$ \\
MFCC + Phon./Artic. & $78 \%$ & 0.78 & 0.871 \\
MFCC + Prosody & $78 \%$ & 0.78 & 0.878 \\
\hline MFCC + Artic. + Phon./Artic & $\mathbf{8 2 \%}$ & $\mathbf{0 . 8 2}$ & $\mathbf{0 . 8 9 7}$ \\
MFCC + Artic. + Prosody & $\mathbf{8 4 \%}$ & $\mathbf{0 . 8 4}$ & $\mathbf{0 . 9 0 0}$ \\
\hline All feature sets & $\mathbf{8 2 \%}$ & $\mathbf{0 . 8 2}$ & $\mathbf{0 . 8 9 5}$
\end{tabular}

for three tasks even for $N=4$; as it meant 104-176 attributes for each subject, we achieved relatively high classification performance even with this compact representation.

\section{Classifier Combination}

For i-vectors the straightforward 'classification' approach is to compare the i-vector of the test speaker with the reference ivector by taking the cosine distance. This approach has a solid mathematical basis and it tends to perform well in practice but it makes the predictions hard to combine with other methods or feature sets. Here, we used a standard SVM, which generates class-wise posterior estimates that provide a simple way of classifier combination by taking the mean of two or more posterior vectors (late fusion [28]).

We will demonstrate the effectiveness of this strategy with two short examples. Instead of applying more classification algorithms, we will focus on combining the different feature sets and tasks. We will apply late fusion by taking the weighted mean of the posterior estimates with an increment of 0.05 ; and similar to our earlier experiments, weights are determined in a nested cross-validation process. We choose the feature sets or tasks by applying the Sequential Forward Selection (SFS, $[29,30])$ approach. First we start with the feature set/task that has the highest metric value. Then we try adding all the remaining feature sets/tasks one by one, and select the one that leads to the highest improvement in the AUC score. Values exceeding the initial feature set/task are shown in bold.

\subsection{Results with Feature Set Combination}

Table 2 shows the results obtained for the Monologue task. Note that the results regarding the MFCC feature set improve when articulatory features are added: the classification accuracy rose from $80 \%$ to $84 \%$, the corresponding $F_{1}$ value went up from 0.8 to 0.84 , and the AUC value of the PD class also rose from 0.880 to 0.908 . However, adding more feature sets proved futile: although the accuracy and F-measure values remained constant even after utilizing the prosodic features as well, the AUC score fell to 0.900 . Still, the 0.908 score achieved by fusing the predictions got from the first two feature sets brought an improvement of $20 \%$ in terms of the RER.

\subsection{Results with Task Set Combination}

Table 3 lists the accuracy, F-measure and AUC scores we obtained when combining the posterior estimates for the different speech tasks besides articulatory features. We got the highest results on the 'Read text' task. It actually matched the performance of MFCCs in terms of the AUC, while the accuracy and $F_{1}$ values appeared to be higher. Besides 'Read text', using
Table 3: Results obtained when combining the different tasks for the articulatory features

\begin{tabular}{l||c|c|c} 
Features & Acc. & $F_{1}$ & AUC \\
\hline \hline Read text & $80 \%$ & 0.80 & 0.848 \\
\hline Read text + DDK & $76 \%$ & 0.76 & $\mathbf{0 . 8 6 0}$ \\
Read text + Monologue & $\mathbf{8 4 \%}$ & $\mathbf{0 . 8 4}$ & $\mathbf{0 . 8 7 8}$ \\
Read text + Sentences & $74 \%$ & 0.74 & $\mathbf{0 . 8 6 2}$ \\
\hline Read text + Monol. + DDK & $\mathbf{8 2 \%}$ & $\mathbf{0 . 8 2}$ & $\mathbf{0 . 8 9 2}$ \\
Read text + Monol. + Sentences & $76 \%$ & 0.76 & $\mathbf{0 . 8 6 7}$ \\
\hline All tasks & $80 \%$ & 0.80 & $\mathbf{0 . 8 7 7}$
\end{tabular}

the 'Monologue' task resulted in a performance improvement, while incorporating the 'DDK' task as well increased the AUC value even further (although the classification accuracy and $F_{1}$ dropped slightly), leading to a $29 \%$ of RER score.

Overall, we achieved significant improvements in both cases by training SVMs for the task-feature set pairs independently, and taking the weighted mean of the posterior estimates. The combination of weights were determined in nested crossvalidation, so it was free of peeking. Our results indeed confirm the flexibility of FV representations. For state-of-the-art performance, it might worth combining different classifiers as well.

\section{Conclusions}

Parkinson's Disease, a chronic neuro-degenerative disease, is often difficult to diagnose accurately. A non-invasive and promising procedure for assessing and diagnosing Parkinson's is the automatic analysis of speech of the subject. Our study showed how useful are FV over i-vectors as features in the assessment of PD via the analysis of speech. We used the PCGITA dataset to classify PD and HC subjects. Samples comprising such dataset were segmented, and cepstral, articulatory, phonological and prosodic features were extracted from the voiced parts. These features were represented by FV-encoding and were classified using Support-Vector Machines. This workflow produced a high-precision classification performance.

The first experiments revealed that MFCC features performed the best in three of the four tasks. The task 'Sentences' became the leader in terms of the AUC, with a score of 0.891. In the subsequent experiments, we showed that the predictions obtained for the different frame-level feature sets and tasks could be combined, allowing an even higher classification performance. This way, our AUC scores improved even further, and we got 0.908 with the combination of MFCCs with articulatory features for the 'Monologue' task, while using the articulatory features, but incorporating the predictions for the tasks 'Read text', 'Monologue' and 'DDK', also led to a significant improvement over relying on the 'Read text' task only. Using different feature sets and/or tasks is not the only possible combination approach possible. Another promising line of research is to apply other machine learning methods, and combine their predictions. This, however, is the subject of future works.

\section{Acknowledgements}

This research was partially funded by the Ministry of Human Capacities, Hungary (grant 20391-3/2018/FEKUSTRAT). This work was also funded by COD at UdeA grant \# PRG2015-7683. G. Gosztolya was also funded by the János Bolyai Scholarship of the Hungarian Academy of Sciences. 


\section{References}

[1] L. V. Kalia and A. E. Lang, "Parkinon's disease," The Lancet, vol. 386, no. 4, pp. 896-912, 2015.

[2] S. Pinto, R. Cardoso, J. Sadat, I. Guimarães, C. Mercier, H. Santos, C. Atkinson-Clement, J. Carvalho, P. Welby, P. Oliveira et al. "Dysarthria in individuals with Parkinson's disease: a protocol for a binational, cross-sectional, case-controlled study in French and European Portuguese (fralusopark)," BMJ open, vol. 6, no. 11, p. e012885, 2016

[3] D. G. Theodoros, G. Constantinescu, T. G. Russell, E. C. Ward, S. J. Wilson, and R. Wootton, "Treating the speech disorder in Parkinon's disease online," Journal of Telemedicine and Telecare, vol. 12, no. 3_suppl, pp. 88-91, 2006.

[4] A. Satt, R. Hoory, A. König, P. Aalten, and P. H. Robert, "Speechbased automatic and robust detection of very early dementia," in Proceedings of Interspeech, Singapore, 2014, pp. 2538-2542.

[5] M. Shahbakhi, D. T. Far, E. Tahami et al., "Speech analysis for diagnosis of Parkinson's disease using genetic algorithm and support vector machine," Journal of Biomedical Science and Engineering, vol. 7, no. 4, pp. 147-156, 2014.

[6] G. Gosztolya, V. Vincze, L. Tóth, M. Pákáski, J. Kálmán, and I. Hoffmann, "Identifying Mild Cognitive Impairment and mild Alzheimers disease based on spontaneous speech using ASR and linguistic features," Computer, Speech \& Language, vol. 53, no. Jan, pp. 181-197, 2019.

[7] L. Zhou, K. C. Fraser, and F. Rudzicz, "Speech recognition in Alzheimer's disease and in its assessment." in INTERSPEECH, 2016, pp. 1948-1952.

[8] L. Moro-Velázquez, J. A. Gómez-García, J. I. Godino-Llorente, J. Villalba, J. R. Orozco-Arroyave, and N. Dehak, "Analysis of speaker recognition methodologies and the influence of kinetic changes to automatically detect Parkinon's disease," Applied Soft Computing, vol. 62, pp. 649-666, 2018.

[9] T. S. Jaakkola and D. Haussler, "Exploiting generative models in discriminative classifiers," in Proceedings of NIPS, Denver, CO, USA, 1998, pp. 487-493.

[10] B. Schölkopf, J. C. Platt, J. Shawe-Taylor, A. J. Smola, and R. C. Williamson, "Estimating the support of a high-dimensional distribution," Neural Computation, vol. 13, no. 7, pp. 1443-1471, 2001

[11] D. G. Lowe, "Distinctive image features from scale-invariant keypoints," International Journal of Computer Vision, vol. 60, no. 2 pp. 91-110, 2004

[12] X. Peng, L. Wang, X. Wang, and Y. Qiao, "Bag of visual words and fusion methods for action recognition: Comprehensive study and good practice," Computer Vision and Image Understanding, vol. 150 , pp. 109-125, 2016.

[13] P. J. Moreno and R. Rifkin, "Using the Fisher kernel method for web audio classification," in Proceedings of ICASSP, Dallas, TX, USA, 2010, pp. 2417-2420.

[14] Y. Tian, L. He, Z. yi Li, W. lan Wu, W.-Q. Zhang, and J. Liu, "Speaker verification using Fisher vector," in Proceedings of ISCSLP, Singapore, Singapore, 2014, pp. 419-422.

[15] Z. Zajíc and M. Hrúz, "Fisher Vectors in PLDA speaker verification system," in Proceedings of ICSP, Chengdu, China, 2016, pp. 1338-1341.

[16] H. Kaya, A. A. Karpov, and A. A. Salah, "Fisher Vectors with cascaded normalization for paralinguistic analysis," in Proceedings of Interspeech, 2015, pp. 909-913.

[17] J. Sánchez, F. Perronnin, T. Mensink, and J. Verbeek, "Image classification with the Fisher Vector: Theory and practice," International Journal of Computer Vision, vol. 105, no. 3, pp. 222-245, 2013.

[18] F. Perronnin and C. Dance, "Fisher kernels on visual vocabularies for image categorization," in 2007 IEEE Conference on Computer Vision and Pattern Recognition, June 2007, pp. 1-8.
[19] M. Seeland, M. Rzanny, N. Alaqraa, J. Wäldchen, and P. Mäder, "Plant species classification using flower images: A comparative study of local feature representations," PLOS $O N E$, vol. 12, no. 2, pp. 1-29, 02 2017. [Online]. Available: https://doi.org/10.1371/journal.pone.0170629

[20] J. R. Orozco-Arroyave, J. D. Arias-Londoño, J. F. Vargas-Bonilla, M. C. Gonzalez-Rátiva, and E. Nöth, "New Spanish speech corpus database for the analysis of people suffering from Parkinson's Disease," in Proceedings of LREC, Reykjavik, Iceland, May 2014, pp. 26-31.

[21] N. García, J. R. Orozco-Arroyave, L. F. D’Haro, N. Dehak, and E. Nöth, "Evaluation of the neurological state of people with Parkinon's disease using i-vectors," in INTERSPEECH, 2017.

[22] P. Boersma, "Accurate short-term analysis of the fundamental frequency and the harmonics-to-noise ratio of a sampled sound," in IFA Proceedings 17, 1993, pp. 97-110.

[23] J. R. Orozco-Arroyave, J. Vásquez-Correa, F. Hönig, J. D. AriasLondoño, J. Vargas-Bonilla, S. Skodda, J. Rusz, and E. Nöth, "Towards an automatic monitoring of the neurological state of Parkinon's patients from speech," in Proceedings of ICASSP. IEEE, 2016, pp. 6490-6494.

[24] N. Dehak, P. Dumouchel, and P. Kenny, "Modeling prosodic features with joint factor analysis for speaker verification," IEEE Transactions on Audio, Speech, and Language Processing, vol. 15, no. 7, pp. 2095-2103, 2007.

[25] A. Vedaldi and B. Fulkerson, "Vlfeat: An open and portable library of computer vision algorithms," in Proceedings of the 18th ACM international conference on Multimedia. ACM, 2010, pp. 1469-1472.

[26] D. C. Smith and K. A. Kornelson, "A comparison of Fisher vectors and Gaussian supervectors for document versus non-document image classification," in Applications of Digital Image Processing $X X X V I$, vol. 8856. International Society for Optics and Photonics, 2013, p. $88560 \mathrm{~N}$.

[27] C.-C. Chang and C.-J. Lin, "LIBSVM: A library for support vector machines," ACM Transactions on Intelligent Systems and Technology, vol. 2, pp. 1-27, 2011.

[28] B. Schuller, S. Steidl, A. Batliner, S. Hantke, E. Bergelson, J. Krajewski, C. Janott, A. Amatuni, M. Casillas, A. Seidl, M. Soderstrom, A. S. Warlaumont, G. Hidalgo, S. Schnieder, C. Heiser, W. Hohenhorst, M. Herzog, M. Schmitt, K. Qian, Y. Zhang, G. Trigeorgis, P. Tzirakis, and S. Zafeiriou, "The INTERSPEECH 2017 computational paralinguistics challenge: Addressee, Cold \& Snoring," in Proceedings of Interspeech, Stockholm, Sweden, Aug 2017, pp. 3442-3446

[29] M. Last, A. Kandel, and O. Maimon, "Information-theoretic algorithm for feature selection," Pattern Recognition Letters, vol. 22, no. 6-7, pp. 799-811, 2001.

[30] I. A. Basheer and M. Hajmeer, "Artificial neural networks: fundamentals, computing, design, and application," Journal of microbiological methods, vol. 43, no. 1, pp. 3-31, 2000. 